\section{Pragmáticas de la sobrevivencia. Un estudio de caso argentino sobre las violencias pre/post carcelarias y el accionar de las mujeres}

\author{
Pragmatics of survival. An Argentine case study on pre/post prison violence and the actions of \\ women
}

María Florencia Actis; Juliana Inés Arens

\section{RESUMEN}

El trabajo busca repensar las trayectorias de mujeres que han sido privadas de la libertad, y los significados diferenciales que asumen sus estadías carcelarias, evitando reinscribirlas en posiciones de "víctimas perfectas". Para ello, se tienen en cuenta no sólo las condiciones estructurales de opresión, y las violencias que de ellas se desprenden, sino también las acciones y decisiones que estas mujeres llevan adelante para sobrevivir. Se presenta el relato de vida de Candela, quien atravesó el sistema penitenciario en dos oportunidades, y se lo analiza a partir de su relación con las agencias punitivas (no penitenciarias) y con la institución familiar, respectivamente. Se realizó una entrevista biográfico-narrativa, en su vivienda, ubicada en la localidad bonaerense de Mar del Plata (Argentina) en el mes de agosto de 2017. Su testimonio permite problematizar, por un lado, el entramado de instituciones que disciplinan las biografías de las mujeres insertas en contextos de pobreza y marginalidad, pero también los "cálculos" y estrategias que ellas mismas despliegan en pos de su cuidado/defensa, y el de sus hijos/as. Se concluye que las mujeres sobreviven a "otras cárceles" que anteceden y se constelan con el sistema penitenciario, dotando a este último de sentidos nuevos y paradojales.

Palabras clave: Mujeres; Itinerarios; Prisión; Violencia de género; Resistencia a la opresión

\section{ABSTRACT}

The article rethinks imprisoned women's trajectories and the differential meanings that their time in prison assumes, avoiding reinscribing them in the position of "perfect victims". That is, these trajectories take into account not only the structural conditions of oppression, and the violence that arises from them, but also the actions and decisions that these women carry out to survive. In this sense, the article focuses on the life story of Candela, a woman who went through the prison system on two occasions, and whose relationship with punitive agencies (not prisons) and with the family institution is analyzed. A biographical-narrative interview was conducted at her home, located in the city of Mar del Plata (Argentina) in August 2017. Her testimony reflects, on the one hand, the network of institutions that discipline the biographies of women in contexts of poverty and marginality, but also the "calculations" and strategies that they themselves deploy in pursuit of their care/defense, and that of their children. It is concluded that women survive "other prisons" that precede and are constellated with the prison system, endowing the latter with new and paradoxical meanings.

Keywords: Women; Itineraries; Prison; Gender violence; Resistance to oppression
RELIGACIÓN

REVISTA DE CIENCIAS SOCIALES Y HUMANIDADES JOURNAL OF SOCIAL SCIENCES AND HUMANITIES
REVISTA DE CENCIAS SOCIAIS E HUMANAS

NFORMACIÓN:

http://doi.org/10.46652/rgn.v6i30.859 ISSN $2477-9083$

Vol. 6 No. 30, 2021. e210859 Quito, Ecuador

Enviado: octubre 04, 2021

Aceptado: noviembre 28, 2021

Publicado: diciembre 15, 2021

Publicación Continua

Sección Dossier | Peer Reviewed
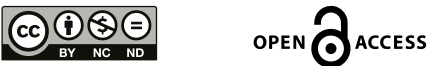

\section{AUTORAS:}

(D) María Florencia Actis

Universidad Nacional de Mar del Plata - Argentina

florenciactis@gmail.com

(D) Juliana Inés Arens

Universidad Nacional de Mar del Plata - Argentina

arensjuliana@gmail.com

\section{Conflicto de intereses}

Las autoras declaran que no existe conflicto de interés posible.

Financiamiento

No existió asistencia financiera de partes externas al presente artículo.

Agradecimiento

A Candela y a todas las mujeres privadas de su libertad y liberadas con las que hemos conversado a lo largo de estos años

Nota

La entrevista que conforma el corpus del artículo fue realizada en el marco de la tesis doctoral de la autora, María Florencia Actis, titulada "Contextos carcelarios y performances de género. Experiencias entre la disciplina y la resistencia sociosexual". Si bien se retoman algunos fragmentos de la entrevista ya publicados en la tesis, se analizan con mayor profundidad y a la luz de nuevas preguntas y ejes de codificación.

\section{ENTIDAD EDITORA}




\section{Introducción}

\subsection{Antecedentes conceptuales}

El estudio de las mujeres y el sistema penal comenzó a adquirir un marco de comprensión más amplio, multi-dimensional, a partir de la década de 1970, con el impulso de los Estudios de las Mujeres y de Género, y el desarrollo de teorías criminológicas feministas en países anglosajones (Coppa, 2019). Dentro de este campo emergieron áreas o líneas de trabajo que abordaron la relación de las mujeres, por un lado, con la institución judicial y los procesos selectivos de criminalización, y, por el otro, con la victimización de las mujeres en diferentes ámbitos sociales, particularmente en los vínculos familiares o de pareja (Haywood \& Mac an Ghaill, 2003). De este modo, el encarcelamiento femenino será repensado en clave de género, y los propios mecanismos de las instituciones penales como instancias nodales en la (re)producción de diferencias de género (Antony, 2007).

En el caso de Latinoamérica, los estudios criminológicos con perspectiva de género feminista se desarrollaron tardíamente, registrándose una mayoría de aquellos centrados en la victimización y el maltrato hacia las mujeres (Beltrán, 2010). Como señala Ileana Arduino (2019), se estableció una correlación entre dimensiones del control en la esfera doméstica y la esfera pública/institucional. Por su parte, en Argentina, los trabajos recientes de Natalia Ojeda (2013), Laurana Malacalza (2015) e Irma Colanzi (2018) se sitúan en diversos aspectos de las experiencias femeninas dentro de las tramas de la gobernabilidad penitenciaria, desde una perspectiva integral que mira estas correlaciones de violencias entre el afuera/adentro. El trabajo actual, en articulación con dichos abordajes, propone un camino inverso, focalizado en las trayectorias previas al encarcelamiento, para comprender desde allí, los significados variables e imprevistos que puede asumir el paso por una cárcel.

A su vez, se inscribe en una perspectiva teórico-política interseccional (Crenshaw, 1991) que supone mirar la gestión del castigo, y por ende la distribución de la vulnerabilidad (Butler, 2009) entre la diáspora de mujeres, y de sujetos marginalizados. En este sentido, se retoma el concepto de "selectividad sexual del sistema penal" (Sánchez, 2012) para actualizarlo desde un enfoque interseccional -y no sólo sexual- que dé cuenta de la especificidad de las vidas capturadas penalmente. Lo que subyace a la retícula de instituciones y discursos penales es una concepción de sexualidad bajo el modelo heteronormativo, articulada históricamente con modelos de racialización de la clase social, que vigilan, re-victimizan y condenan la sexualidad y el género de las mujeres pobres (Davis, 2005; Almeda Samaranch \& Di Nella, 2017; Senatore, 2019). En términos de Almeda Samaranch:

...la exclusión de la exclusión es el perfil que sobresale cuando se estudia a las mujeres encarceladas, porque muchas de ellas ya fueron excluidas socialmente antes de estar condenadas y, una vez encerradas, la cárcel las vuelve a excluir con sus políticas de aprisionamiento y castigo (2017, p. 157).

Hablamos de trayectorias mayormente signadas por opresiones y privaciones de derechos básicos, que anteceden a la prisión. El informe del Centro de Estudios Latinoamericanos sobre 
Inseguridad y Violencia (Bologna et al., 2019), llevado a cabo en centros penitenciarios de ocho países latinoamericanos (Argentina, Brasil, Chile, Costa Rica, El Salvador, Honduras, México y Perú), arroja que las trayectorias educativas de las mujeres detenidas en la región se interrumpen mayormente antes de egresar del nivel secundario, y representan una mayor proporción de fuerza desempleada que los varones antes de su encarcelación. A su vez, el $55 \%$ de las mujeres tuvo su primer/a hijo/a antes de los 18 años, porcentaje que se reduce a $26 \%$ para el caso de los varones. Por último, el número de mujeres que dice sentirse más segura dentro que fuera de la cárcel supera al de los varones. La "libertad" de las mujeres pobres, está colmada de otras expresiones/ prácticas de disciplinamiento y violencia social, que en lo cotidiano se alejan de la realidad de sus compañeros detenidos. Pero también difieren (radicalmente) de las experiencias de violencia de género que atraviesan las vidas de mujeres no criminalizadas, valiendo preguntarse ¿qué tienen en común los padecimientos de unas y otras?, ¿̇o las formas en que son interpeladas socialmente como mujeres?, ¿alcanza el género como variable para su análisis? Como plantea Arduino:

Sigue pendiente en la criminología feminista, el desafío de poder producir un conocimiento desde las distintas experiencias de opresión de las mujeres donde el género sea una variable más junto con la raza, la clase, la sexualidad, la edad, entre otras. De esta forma, la interseccionalidad consiste en construir una criminología feminista que repare en que las relaciones de género no ocurren en el vacío (Iglesias Skulj citada por Arduino, 2019, pp. 125-126).

\subsection{Repolitizar la violencia, rearticular el género}

Frente a la extensión del concepto de violencia a diversas áreas de lo social, y a la pérdida de su poder explicativo, se torna necesario menos insistir en la búsqueda de una definición exacta, que contextualizar dicho proceso, y señalar implicaciones en materia de género.

En primer lugar, se plantea que la violencia no es un comportamiento incivilizado o anómalo que irrumpe en la sociedad -imaginada como naturalmente libre de conflictividad-, sino que debe ser abordada desde su carácter ambiguo y paradojal, como dimensión constitutiva de las experiencias sociales, cumpliendo una función social productiva y destructiva a la vez (Garriga Zucal \& Noel, 2010).

Los investigadores de la 'violencia' debemos hacer el esfuerzo por restituir a la 'violencia' su justo lugar en la normalidad de lo cotidiano y lo habitual para no olvidar tener en cuenta, a la hora del análisis, su papel positivo en el origen, sostén y refuerzo del lazo social, por más que todos nuestros reflejos intelectuales conspiren para que lo olvidemos (p. 100).

El ingreso de la violencia de género como tema mainstreaming de las agendas públicas en Latinoamérica -y el mundo-, a partir de 1980, ocurre en un contexto social más amplio de apuesta al institucionalismo y a la penalidad como vía legítima (y pacífica) de resolución de conflictos. Tamar Pitch (2014) propone pensar estas nuevas posibilidades del género a partir de un cambio de paradigma epistemológico que se expresa en una mutación del lenguaje de la opresión al de la violencia. 
Nombrar diversas conductas que antes se entendían como producto de sistemas de opresión en términos de 'violencia' permite establecer una clara diferencia entre víctima y agresor, así como individualizar responsabilidades, lo cual nos lleva a los terrenos de la justicia penal (Pitch citada por Núñez Rebolledo, 2019, p. 74).

En este sentido, la codificación de las opresiones sociales y de género bajo el lente de la violencia se produce por/en la emergencia de lo que Pitch denomina una "sociedad de víctimas", es decir, "la modelación de la escena social conforme a la escena penal, y de la escena penal conforme a una fuerte vocación moralizante” (2014, p. 23). La violencia de género, su tipificación como delito y su inscripción en el campo de la criminalística, al igual que su solución de la mano de la prevención, no hizo más que sustraer la violencia de una dinámica social estructural y reforzar una gramática generizada que rubricó - una vez más- a las feminidades como lugares esenciales de temor e indefensión (Marcus, 2002).

Si bien la categoría víctima puede resultar particularmente útil para el caso de las mujeres detenidas, o que han atravesado procesos de encarcelamiento, a los fines de visibilizar su situación, se torna fundamental no aislar, y circunscribir, la problemática de la violencia de género, para entender las dimensiones y modulaciones que adquiere en contextos de desposesión estructural. Pero también para evitar que la sobre-representación de la víctima haga sombra a las luchas y resistencias cotidianas de las mujeres (Aguirre \& Beltrán, 2016).

Es importante en este punto señalar (y eludir) la tendencia hacia una construcción dicotómica y excluyente entre ambas condiciones, el ser víctima y el ser agente. Tal como plantean Diego Zenobi y Maximiliano Marentes (2020), en el marco de esta tendencia, la figura de la "víctima" representaría la disminución par excellence de la agencia (p. 69) connotando una clara "identidad negativa" (Gatti, 2016) que impediría el ejercicio pleno de ciudadanía y personificaría una manifestación del ser humano (sólo) en situación doliente. A contrapelo de esta lectura, se concibe no sólo la irreductible capacidad de agencia y una síntesis posible "víctima-agente" (Roggeband, 2010), sino al propio devenir-víctima como una forma contemporánea, especialmente efectiva, de agenciamiento y activismo. En términos de Gabriel Gatti (2016), "la condición de víctima constituye actualmente uno de los catalizadores más intensos de solidaridad social; ésto es, las víctimas son uno -aislados, solos, incomunicados- y son también multitud" (p. 118). De este modo, ser víctimas no cancela a priori la acción individual/política, pero se inscribe casi indefectiblemente en el repertorio discursivo de las violencias, "los traumas” y las soluciones/reparaciones individuales.

Si bien a lo largo del trabajo se tendrá en cuenta la noción de víctima-agente por constituir una categoría nodal que interpela las luchas de las mujeres y sus posibilidades concretas de reconocimiento hoy, se va a privilegiar la esencia del concepto de "subjetividad activa y resistente" propuesto por María Lugones (Abellón, 2014), en tanto da cuenta con mayor precisión de las formas de hacer, gestionar y resolver de ciertas mujeres, ya no frente a "hechos de violencia" sino ante condiciones permanentes de opresión. El concepto es referido por Lugones como "una activación en cuanto a las maneras de ser, pensar y practicar que para uno/a son constitutivas” ( $p$. 188), y que usualmente no derivan en expresiones mayúsculas de acción social o militancia política - puntos de cristalización de la agencia - , sino que comprenden más bien formas infra-políticas de agencia, pragmáticas, desestructuradas o no estructuradas "dentro de algo", subrepticias, y 
por tanto, invisibles a los ojos del poder, no hegemónicas. Una resistencia/potencia, subjetiva y corpórea, en tanto nunca es ni existe desapegada de su lugar de arraigo: el cuerpo que la contiene (Gago, 2019).

En esta dirección, el trabajo no busca constatar qué tan víctimas son las mujeres pobres y criminalizadas, sino abrir preguntas en relación a la utilidad y márgenes de resignificación/ reapropiación de la noción de víctima, como así también conocer las formas en que se pone en juego y se activa una subjetividad de la resistencia y la sobrevivencia, eludiendo la tendencia a su fijación y absolutización en posiciones de víctimas pasivas, de "víctimas perfectas". En otras palabras, se busca renovar el enfoque victimo-céntrico que ha predominado en la construcción de sus genealogías de vida, para politizar los entornos de socialización, pero también sus acciones y decisiones.

\subsection{Objetivos}

Se plantea como primer objetivo narrar la trayectoria de vida de "Candela" a partir de dos ejes de codificación y análisis: las instituciones punitivas, o lo que Michel Foucault denominó "dispositivos de encarcelamiento múltiple" (2015), y la familia heterosexual, que comprende a la familia nuclear de la infancia y, sobre todo, a su relación de pareja conformada en la etapa joven-adulta. El abordaje desde estas dimensiones nos permite acceder al entramado de instituciones disciplinarias, formales e informales, presentes en su trayectoria para "intentar sacar una 'fotografía' que muestre el ejercicio permanente de la violencia” (Herrasti Maciá, 2010, p. 35), pero también que reconozca decisiones/acciones en clave de agenciamientos, y de la dialéctica violencia/resistencia. El segundo y último objetivo, es problematizar los significados paradojales (Ojeda, 2013) que puede asumir la cárcel para el caso específico y situado de las mujeres. El propósito general es explorar y entrelazar dimensiones macro-micro políticas que constituyen las vidas de quienes se encuentran detenidas.

\section{Metodología}

El trabajo se inscribe en una investigación cualitativa, en tanto se priorizan los significados que las mujeres construyen (y disputan) en torno a sus propias vivencias. En este marco, se reflexiona a partir de una entrevista biográfica sobre un caso particular relatado en primera persona por una mujer, a quien llamaremos "Candela". Consideramos que trabajar esta problemática mediante un estudio de caso y una entrevista biográfica posibilita desestabilizar las representaciones hegemónicas sobre las mujeres privadas de su libertad, e imprimir corporalidad a las voces que se auto-relatan (Stephen, 2015). A pesar de que es posible entender ciertos contextos y experiencias como susceptibles de ser extendidos a otras mujeres que han pasado situaciones similares a las de Candela, desde ya, no es posible generalizar sus prácticas ni tomar su caso como un modelo paradigmático.

En concreto, el relato biográfico nos permite conocer una "textura detallada" (Lozares \& Verd, 2008) de la vida de Candela, a través de la cual es posible acceder a una gran cantidad de información que sitúa espacio-temporalmente vínculos y procesos entre acontecimientos, actores sociales, valoraciones y emociones. Es decir, reponer y comprender su trayectoria de 
vida (y de encarcelamiento) en clave holística, identificando allí una experiencia social/subjetiva, ineluctablemente arraigada en lo corporal/emocional. Además, al tratarse de una narración necesariamente selectiva, fue factible reconocer ciertos "núcleos temáticos" (Lozares \& Verd, 2008) de particular interés para Candela, cruciales para entender cómo fueron vividos los acontecimientos e influyeron en su toma de decisiones.

En este sentido, el abandono como una forma invisibilizada de violencia, emerge como un primer núcleo o estructura de sentido en su relato, que conecta diversas situaciones, agentes y etapas de su vida, y frente al cual se vislumbra un modo de ser, de hacer y de simbolizar la propia vida. Por otro lado, ocupa un lugar preponderante lo que describe como un sistema de corrupción de las instituciones penales/policial, no vinculado con actitudes aisladas de funcionarios aislados, sino contrariamente, con un mecanismo de funcionamiento naturalizado, a partir del cual irá adquiriendo y desplegando un saber hacer, un aprendizaje estratégico, que va a permitirle atravesar y sortear determinados contextos. Por último, en relación con el abandono y las violencias, el cuidado, en un sentido amplio, y referido al rol de cuidar, sobre todo al de ser cuidada (por una pareja, una institución, una red feminista) representa una dimensión constitutiva de sus expectativas/demandas respecto de los otros/as. Es decir, a través del reconocimiento de estos núcleos temáticos y de sentido, trazados por la propia Candela, nos acercamos a procesos de subjetivación más amplios, que nos ofrecen no sólo una perspectiva subjetiva de la entrevistada sino también ético-política, en tanto conocemos aquello que es considerado socialmente justo, valorable y necesario para ella misma, y para otros/as.

A su vez, concebimos que una metodología centrada en una entrevista de tipo biográfica favorece al reconocimiento de la interdependencia entre procesos macrosociales, caracterizados por profundas desigualdades en el acceso a la justicia, y experiencias personales en términos de proceso (Scott, 1992). En cuanto al eje de reflexión sobre el sistema carcelario, permite visualizar las violencias pre/post carcelarias, las afectaciones múltiples en la subjetividad de las personas que pasan por la institución y los modos en que dinámicas intra-muros se articulan con diversos niveles y dimensiones de la vida extra-muros, pudiendo dar cuenta de la porosidad de los límites carcelarios.

En cuanto a la elección del caso de Candela, ésta responde a que, además de su paso por el encierro penitenciario, porta la experiencia de la institucionalización a temprana edad y el haber estado en situación de calle. Este itinerario, a la luz del análisis, se convierte en un factor posibilitador tanto de los reiterados contactos con el sistema penal/asistencial, y con el ambiente delictivo, como también de la construcción de un saber hacer particular sobre los modos de interactuar con el sistema penal y sus distintos actores.

Por otra parte, es necesario tener en cuenta que aquello que presentamos como "resultados" de investigación, no debe ser tomado como el reflejo simple y neutral de una problemática, sino como un saber inscripto en un contexto discursivo de producción académica. El relato biográfico de Candela es un discurso elaborado y mediado a través de un proceso de investigación, y concretamente de una situación/escena de entrevista, configurada a partir de relaciones de poder/saber. 
En primer lugar, la toma de la palabra no surge en Candela como un acto voluntario, libre o espontáneo, sino que responde al interés de quienes propiciamos la investigación, solicitamos el relato, y por ello, participamos del mismo en calidad de co-autoras (Cruz Contreras, 2018), haciendo de la escucha activa, del registro/grabación de su palabra y de nuestras intervenciones, condiciones que afectarán irremediablemente "lo decible” y “lo dicho". El conocimiento que funge de dicha escena supone una práctica de poder, de negociación, de articulación y de subjetivación entre quien entrevista - y traza los marcos de enunciabilidad - y quien elige responder - o no -. En el caso de Candela, más allá de las preguntas que han guiado, y constreñido, la conversación, es de destacar la extensión en sus respuestas, la necesidad voraz de contar su historia, de decir su palabra, pudiendo concluir que este encuentro en particular, a partir de las características de Candela - una mujer que vive sola con sus hijos/as, todavía niños/as, sin amigas ni redes de contención - se ha configurado como un espacio de interlocución regido por condiciones de expresión y enunciación de su palabra, menos restrictivas que posibilitadoras.

En segundo lugar, durante la instancia de desgrabación de la entrevista, procesamiento y análisis a la luz de categorías y ejes de codificación, pero también de un proceso colectivo de escritura, tuvo lugar una nueva mediación y construcción narrativa. De esta manera, consideramos que el relato final condensa un conocimiento situado, encarnado (Esteban, 2004) y parcial, que resulta de sujetos, posicionamientos, procesos e interacciones varias, cuya visualización, y actual planteamiento, nos acerca justamente a un conocimiento más riguroso, responsable, específico y - sólo en este sentido - objetivo (Haraway 1995).

En pos de hacer visible nuestro propio lugar de enunciación, vale mencionar que el trabajo se apoya en recorridos de campo más amplios, y en diversos proyectos de investigación académica, que han incluido etnografía en contextos penitenciarios de mujeres y de sus hogares, barrios y comunidades de residencia. Puntualmente, el contacto con Candela y la entrevista en cuestión tuvieron lugar durante el proceso de tesis doctoral de una de las autoras del texto, desarrollada entre los años 2015 y 2019 en la Unidad Penitenciaria Nº 8, dependiente del Servicio Penitenciario Bonaerense (SPB). En este sentido, buscamos dialogar con aquellos estudios etnográficos sobre el tema carcelario que proponen un abordaje integral, pero también feminista, vinculados a un interés en mirar procesos de (de)construcción de la categoría género a través de las interacciones de las mujeres que transitan por el afuera/adentro de la cárcel.

\section{Resultados}

A los fines de conocer y comprender el significado de la prisión en el marco del relato biográfico de Candela, accedemos a la trama de experiencias, sentires y valoraciones en distintas etapas de su vida, de su itinerario. Cada una de ellas involucra diferentes actores e instituciones que producirán determinadas interpelaciones en ella y, en consecuencia, estrategias variables de resistencia y sobrevivencia.

En este sentido, los siguientes apartados están asociados a tres momentos en concreto. El primero, se centra en el relato de su infancia, en tanto es en esa etapa donde ella ubica ciertas "escenas fundacionales", posibilitadoras de los acontecimientos futuros. El segundo, presenta el último tramo de su primera condena (octubre del 2000-julio del 2004) y el tiempo en libertad, una 
etapa afectada de manera transversal por la relación con su ex-pareja y padre de sus cuatro hijos/ as menores, a quien llamaremos José. El modo en que se relatan los acontecimientos y procesos de este momento resulta de central importancia para situar las pragmáticas de la sobrevivencia y, a su vez, para comprender los sentidos que asume la privación de libertad. El tercero y último, desarrolla el modo en el que logró resguardarse de la violencia de su ex-pareja, y en que atravesó su segunda detención carcelaria (enero del 2009-diciembre del 2009).

\section{1. "Me robaron la posibilidad de la infancia"}

La entrevista tuvo lugar el 5 de agosto del 2017. Candela tenía 39 años al momento del encuentro y cuatro hijos/as, de entre 9 y 12 años. Vivían los/as cinco en una casilla, a unos 45 minutos del centro de Mar del Plata - ciudad costera a $400 \mathrm{~km}$ de la Ciudad Autónoma de Buenos Aires (CABA)-. Contaba con un solo dormitorio y un espacio de no más de 10 metros cuadrados que funcionaba como comedor y daba a la calle. La puerta estaba abierta, y sus hijos/as entraban y salían de manera incesante; estaban jugando al fútbol en la parroquia del barrio. Era sábado por la mañana. Había sol, pero estaba fresco. Candela preparó unos mates y comenzamos a conversar.

Nació en el municipio de Morón, en la zona oeste del área metropolitana de CABA. Se instaló en Mar del Plata en 2009, después de su segunda y última detención carcelaria, como estrategia para escapar de su ex-pareja, quien ejercía violencia sobre ella y la había amenazado de muerte. En el marco de la entrevista nos dijo que no tenía amigas, que no invitaba a nadie a su casa y que no hacía planes nocturnos. Dijo: "Vivo para los niños". Candela tenía ganas de repasar y de compartir su historia. Su relato era minucioso: conectaba experiencias, señalaba responsables (presencias/ ausencias), evaluaba consecuencias, describía un sistema.

\section{F: ¿Cómo definirías al sistema carcelario? \\ C: Yo creo que el abandono vino desde que yo tenía 11 años, cuando me fui de mi casa por ser abusada y el Estado no hizo absolutamente nada.}

Resulta elocuente el modo en que responde a la pregunta sobre cómo definiría ella al sistema carcelario, señalando como un hito en su historia el abuso sexual practicado por su padrastro y al abandono, tanto del Estado como de su madre. En su relato, "el abandono" aparece con frecuencia como una figura específica o solapada de violencia, posibilitadora de otras. Desde entonces, Candela quedó en situación de calle hasta ingresar al Instituto de Menores de Morón, donde volvió a atravesar situaciones de abuso -“de todo tipo"- por parte de funcionarios judiciales.

A mí me robaron la posibilidad de la infancia, yo era una nena. Todavía no era señorita. Pero hubo abandono total de mis padres y de los que tenían que hacerse cargo de esa niña. Si los oficiales que tenían mi causa se acostaban conmigo a los 15 años, ¿qué pude haber aprendido? Uno intentó abusarme en su despacho y, como no quise, me armó una causa por robo y me llevó a un Instituto penal-psiquiátrico. Yo todavía ni delinquía.

Del Instituto se escapó por los techos y, otra vez, hizo de la calle su lugar. En ese momento se intensificó el consumo de drogas que había iniciado al irse de su casa y, junto con el consumo, el robo. Pasó seis años entre la calle y los "hotelitos", hasta que cayó detenida a los 21 años por robo calificado del automotor, agravado por uso de arma. La condenaron a 12 años de prisión que, 
gracias al acceso a beneficios, se redujo a una condena de 3 años y 9 meses. El deterioro físico propio del consumo y la vida en la calle, se profundizó con la detención.

Era una comisaría del menor. Después estuve en una de hombres, en la primera de Hurlingham. Después a la femenina. Yo pesaba 47 kilos y medía 1,70. En ese momento me trasladan a la Unidad 33.

En su relato, Candela narra una infancia atravesada por diversas temporadas de institucionalización penal/asistencial que, además de "robar infancias” y sobre-vulnerar niñeces, producen activamente sujetos/as dotados de ciertos saberes y experiencias, habilidades, identificaciones, demarcando trayectorias marginales, cada vez más alejadas de las prácticas y espacios de ciudadanía. En términos foucaultianos, se trata de una multiplicidad y entrecruzamiento de diversos mecanismos disciplinarios, que ejercen "un discreto poder de castigar" (2015, p. 353). Institutos y tribunales de menores, entidades psiquiátricas, comisarías, toda una red de agencias e instituciones con regímenes particulares de control, por las que niños/as y adolescentes literalmente circulan. Esteban Rodríguez Alzueta enfatiza la tendencia a la circularidad, y propone la metáfora del "círculo" o "circuito" carcelario, para dar cuenta de este "sistema de rotación por diferentes instituciones que van perfilando biografías estigmatizadas y creando las condiciones para ser seleccionados/as por el sistema carcelario" (2015, p. 40). Es decir, trazando una relación de continuidad entre los/as asistidos/as (víctimas) y los/as futuros/as judicializados/as (victimarios/ as).

En el caso puntual de los institutos de menores, instancia en la que se detiene y explaya el relato, pese a la derogación legal del paradigma tutelar en el año 2005, y la formalización de una perspectiva de protección integral de los derechos de los niños, niñas y adolescentes, en la práctica siguen permeadas por el imaginario de sujetos tutelables, incapaces, e irreversiblemente problemáticos. A su vez, en el caso puntual de la provincia de Buenos Aires, estos espacios padecen serios déficits estructurales (edilicios, de hacinamiento, alimentarios, educativos, recreativos, de recursos humanos, etc.) configurándose como "instituciones en crisis" (Axat, 2008, p. 326). Es decir, ofrecen un tratamiento real que, por acción y omisión, termina aumentando la violencia y la situación de marginalidad que, en la teoría, la intervención estatal buscaba evitar.

Ahora bien, sin ánimos de cuantificar o medir el sufrimiento producido de acuerdo al género, quienes adscriben a la identidad Mujer, y además son niñas o adolescentes, son situadas en posiciones inherentemente diferenciales al interior de los juegos de poder que trazan estas instituciones, implicando otro tipo de violencias, y por ello, de negociaciones y estrategias de supervivencia. Poseer un cuerpo feminizado supone habitar un cuerpo especialmente sexualizado. Candela menciona, “yo ya tenía 15 o 16 años, medía 1, 74, era linda, muy flaquita”. Sin embargo, esta diferencia no traduce apriorísticamente al cuerpo en un espacio de dominio/doblegación, quebrado, sino más bien en un espacio de disputa donde la sexualidad, en tanto saber hacer aprendido, puede devenir en un capital, incluso en un saber sobre el cuerpo masculino que, dependiendo del contexto, será plausible de ser utilizado y reconvertido en provecho de una, siempre relativo. En este caso, evitar sanciones, traslados, una causa armada, o simplemente, preservar la existencia. 
En términos generales, a partir/en contra de las experiencias de padecimiento y sobre todo de desprotección -en tanto niña- (que van desde el abuso intrafamiliar hasta la sucesión de encierros y castigos) se vislumbra en Candela un despliegue defensivo/inventivo (Gago, 2019), una pulsión de transgresión y búsqueda de cambio de su situación, materializada en una serie de acciones y decisiones como fugarse del hogar y luego del Instituto, optar por la vida en la calle, rehusarse a realizar determinadas prácticas.

\section{2. José. "Yo quería que muriera, realmente"}

Al poco tiempo de ingresar a la Unidad No 33 -Los Hornos, La Plata-, Candela quedó embarazada luego de un encuentro sexual eventual y fue reubicada en el pabellón de "Madres". Vale mencionar que esta Unidad, enteramente femenina, e inaugurada en 1999, tiene la particularidad de haber sido la primera del SPB en habilitar pabellones de "Madres", destinados al alojamiento de mujeres embarazadas y con hijos/as menores de cuatro años. Antes de cumplir el año en "la 33", Candela protagonizó una denuncia al SPB, en donde reclamaban que este tipo de pabellones permanecieran "desengomados" -abiertos las 24 horas del día-, para que las mujeres detenidas pudieran asistir a sus hijos/as en caso de ser necesario. A último momento, las compañeras se desmarcaron del acuerdo y Candela fue la única que denunció. El penal fue intervenido y se logró la reivindicación; sin embargo, Candela cargó con el castigo ejemplar y fue trasladada junto con su hijo.

“Me trasladaron, me pegaron, me lastimaron. Me bajaron un diente", cuenta. El traslado es una práctica normalizada dentro del sistema penitenciario bonaerense que configura una forma de castigo dentro de las lógicas de la gobernabilidad penal (Daroqui et al., 2014). Los traslados suelen realizarse por la noche y sin dar tiempo a que la persona pueda tomar sus pertenencias, a veces, ni siquiera vestirse. Los grupos familiares y de allegados tampoco son notificados del traslado ni se les informa si se trata de traslados definitivos, obstaculizando así dichos vínculos y la posibilidad de que les sean proveídos los elementos personales y de aseo durante varios días o semanas.

En ese contexto, Candela conoció a José, nueve años mayor, quien también estaba detenido. El contacto se mantuvo únicamente por cartas hasta que a él le otorgaron la libertad.

En un momento me llega una carta y me dice que había recuperado su libertad, que si quería conocer a alguien distinto que lo llamara, y la verdad que la propuesta era interesante. Yo estaba lejísimo de mi casa, sola como un perro, con mi niño, ¿no? Y bueno, lo llamé y me dijo que el fin de semana iba a verme.

José la ayudó a denunciar el amedrentamiento que padecía por parte de funcionarios/as del SPB. Cabe señalar que cuando Candela recuerda el uso sistemático de la violencia durante esa condena, señala: “Querían intervenir los derechos humanos de La Plata, recién se estaba haciendo la organización del Comité contra la Tortura [de la Comisión Provincial por la Memoria]”. En este punto, es posible distinguir un saber hacer, en tanto conocimiento práctico que las personas que transitan el encierro, y sus familiares y allegados, producen en torno al modo de construir denuncias de las violencias institucionales, de constituirse como víctimas de las mismas (Pita, 2019) y reconocer interlocutores válidos, en este caso, el Comité contra la Tortura. 
Volviendo a José, empezó a colaborar en la crianza de su hijo, sacándolo de la cárcel al cumplir los 4 años -edad estipulada por ley para la externación de los/as niños/as-. De hecho, lo reconoció como hijo propio ante el Registro Nacional de las Personas. Al poco tiempo de iniciadas las visitas de José, Candela quedó embarazada y la relación se consolidó.

Me va a ver un año. Me parecía un tipo divino, no faltaba a una visita, respetuoso, caballero. No tenía léxico tumbero, no tenía ningún tatuaje. No sé si me gustaba. Él llegó en el peor momento de mi vida y me había salvado de que me mataran. Y yo soy una piba muy agradecida.

Cuando Candela quedó en libertad, decidieron casarse y convivir. Ella comenzó a trabajar en la economía informal, dedicándose a la venta ambulante de mates, insumos de artesanías y sahumerios. Tenía el taller en su casa, donde armaba los mates, y un puesto de venta al público en la estación de trenes del municipio de Lanús, al sur de CABA. La convivencia resultó buena los primeros dos meses, hasta que Candela se dio cuenta que José consumía cocaína y que, en sus palabras, tenía "psicopatías muy complejas". A su vez, para sostener su consumo, José vendía estupefacientes al menudeo desde la casa compartida con Candela y los/as niños/as.

\begin{abstract}
Después de las 10 de la noche estaba duro atrás de la heladera, y si yo no les vendía a los que venían a comprar, cuando a él a las 2 de la mañana se le pasaba y quería más plata para consumir y no había, me mataba. Me despertaba con el hacha en la nuca. (...) Yo creo que donde hay violencia física, hay violencia psicológica, emocional, económica. No sólo está enfermo él sino que lo mismo te lleva a estar enfermo a vos (...) Me ha hecho cosas muy perversas. Y amén de los abusos, porque hubo abusos sexuales muy complejos. J* [la primera hija con él] ahora tiene 12 años, después nace $D^{*}$ que tiene 11 años, después $\mathrm{V}^{*}$ que tiene 10 y después $\mathrm{M}^{*}$ con 9 . Por eso digo que donde hay abuso físico hay abuso de todo tipo. (...) Tengo 36 denuncias penales por violencia, así que obviamente estuve hospitalizada varias veces, en terapia intensiva, embarazada. Creo que pude soportar esa relación totalmente perversa y enferma porque antes había estado presa. Porque si no hubiera sobrevivido al sistema carcelario, no hubiera sobrevivido a él.
\end{abstract}

Teniendo en cuenta la trayectoria de Candela, su decisión de aceptar verse con José, pareciera estar movida por lo que Verónica Gago denomina una "pragmática vitalista” para referirse a una forma de hacer "de los de abajo" donde "el cuerpo emerge como una memoria de esas cosas que le son útiles, que lo alimentan y lo benefician" (2014, p. 13). En primer lugar, y a sabiendas de lo que significa atravesar la cárcel siendo "paria” o "piedra” para las compañeras de pabellón, Candela encontró en José la posibilidad de recibir visitas, por ende, contención emocional, sustento material y acompañamiento de su causa. A su vez, el vínculo le posibilitó establecer nexos con el afuera. Como se mencionó, fue él quien vehiculizó la denuncia ante el Comité Contra la Tortura sobre la situación de persecución e intimidación que estaba viviendo por parte del SPB. En segundo lugar, la idea de formar una familia no aparece en ningún momento de su relato ligada a la constitución de un proyecto de vida, sino más bien a un modo de gestión y amplificación de la crianza intra-muros, ante la escasez de redes afectivas, recursos económicos y logísticos en el afuera. Una vez excarcelada, sin embargo, el espacio familiar devino en un lugar no sólo improductivo, donde las cargas se distribuyeron inequitativamente, sino en ese infierno que le impedía seguir adelante, poniendo en riesgo su vida y la de sus hijos/as. 
A su vez, cuando comenzaron a recrudecer las situaciones de violencia, la propia experiencia carcelaria fue capitalizada para resistir -emocional y físicamente- a la violencia de género. De este modo, su percepción y vinculación con la violencia de género es desde su condición y su memoria como sobreviviente del sistema carcelario, resultando imposible extraer o separar de su condición de género (del criar, ser madre, ser esposa, ser víctima de violencia o sobreviviente), los saberes y saber haceres construidos en el marco de su paso por la cárcel (el autocuidado, la autodefensa, la reivindicación de sus derechos) y los procesos de institucionalización previos.

Según su relato, José tenía vínculos (negocios) con la comisaría del barrio, por lo que sus denuncias por violencia no prosperaron y él nunca pasó más de 24 hs. detenido. Al no encontrar ninguna respuesta en las instituciones, Candela cuenta que llegó a considerar la posibilidad de asesinarlo.

\begin{abstract}
Había comprado hasta la nafta para prenderlo fuego. Yo decía: "Yo le voy a rociar el acolchado donde él se pone con la pipa, al costadito de la ventana". Digo: "Lo rocío con nafta, abro las hornallas y me voy". Yo quería que muriera, realmente. Y pensaba que algún día lo iba a matar (...) Si yo lo hubiera hecho, hubiera estado detenida por "homicidio agravado por el vínculo, premeditado, con alevosía, ensañamiento" y me hubieran dado 40 años, porque no estaba en ninguna red feminista, ni conocía compañeras que luchan para que se haga justicia, ni absolutamente nada.
\end{abstract}

Como señala Elsa Dorlin (2018), la violencia de las mujeres difícilmente pueda ser vista como legítima defensa, sino como una amenaza, ya que cuanto más se defienden, mayor es la condena frente a su accionar. Dorlin plantea una distinción entre el uso de la categoría jurídica de "legítima defensa” y la simple “autodefensa”. La primera está disponible para aquellos/as sujetos/as previamente construidos y percibidos como sujetos con capacidad legítima de defenderse y de utilizar violencia; mientras que la segunda, le corresponde a aquellos/os otros/as cuya acción defensiva será siempre vista como un exceso, y una práctica subalterna, merecedora de castigo. En palabras de la autora, "la misma posibilidad de defenderse es el privilegio exclusivo de una minoría dominante. (...) A los cuerpos violentables y vulnerables no les corresponde más que subjetividades sin armas en las manos (2018, p. 29).

La vinculación de las mujeres con el ejercicio de la violencia ha sido mayormente imaginada (y estudiada) a partir de su rol como receptoras pasivas, como víctimas y no como perpetradoras. Pero también han quedado claramente excluidas del repertorio de la violencia legítima, ya que su eventual violencia ha sido históricamente patologizada e inscrita en el terreno de las pasiones, de las emociones, y no del ingenio, de la racionalidad. A contrapelo de estas ecuaciones, instauradas desde la criminología positivista, en el caso de Candela, la posibilidad de hacer uso de la violencia forma parte de una acción premeditada, afirmada y hasta deseada: "Yo quería que muriera realmente". De una "necesidad vital en tanto praxis de la resistencia" (Dorlin, 2018, p. 28). E incluso, teniendo conocimiento acerca de cómo el sistema penal interpreta y resuelve frente al accionar violento de una mujer, es que desiste del plan y busca una alternativa con un menor costo para su libertad. 


\section{3. La cárcel como refugio}

La forma que encontró Candela de resguardarse de la violencia, ante la falta de respuesta institucional, fue -en la jerga carcelaria- “fabricarse una causa” y caer detenida nuevamente.

En la última denuncia, yo lo denuncio por venta [de estupefacientes] en un Juzgado Federal. Ellos dicen que me van a acompañar, que me van a contener, y cuando allanaron mi casa fuimos los dos detenidos. O sea, él tira la droga para el vecino de al lado y no le encuentran nada. Así que, si a mí me mataba a palos y yo nunca le había hecho nada, imagínate si se enteraba que lo había denunciado. A los cinco días nos excarcelaban, porque a la droga no la encontraron. Me iba a matar. Él ya sabía que la denuncia la había hecho yo, así que pedí declarar y le dije al fiscal que yo vendía. Mi causa dice: 'Los dichos de autoría de la acusada surgen de su declaración', o sea, nadie decía que yo vendía, solamente yo. La única que se deja presa soy yo misma, pero porque era la única manera de salir de la violencia.

Aquí emerge una nueva instancia de cálculo estratégico por parte de Candela, la auto-detención, en tanto su decisión le supuso volver al contexto de encierro y perder a los niños/as por un tiempo. Sin embargo, asegura “hoy estaría muerta”. Un cálculo en pos de su integridad y la de sus hijos/as, ya que para muchas mujeres ser madres supone disponerse al sacrificio y al riesgo, con tal de protegerlos/as (Salinas Boldo, 2014). Pero también un cálculo imaginable y que sólo cobra sentido en ese contexto tan particular de violencia de género, y en el marco más amplio de su biografía, de su familiaridad con las instituciones penales, y en concreto, con el sistema carcelario. Es decir, esta ingeniería que encuentra como resolución, como salida y como un nuevo modo de fugar, no se agota en la explicación de la mujer desesperada, sino que responde a un trayecto de institucionalización; habla de lo que es conocido para ella.

En su relato, la cárcel representa un lugar, temporalmente, más seguro que su casa. E incluso al ingresar por segunda vez en 2009, con una edad mayor, ya hecha "una doñita", pudo transcurrir, según cuenta, una estadía más tranquila, y formar un grupo "muy genial” de compañeras, como una familia. Es decir, además de funcionar como un refugio, la estadía carcelaria implicó la construcción de vínculos de compañerismo y amistad como formas reconstitutivas del plano emocional subjetivo (Ojeda, 2013), pero también, adquirir conceptos y referentes otros de familia, desplazados/dislocados del hogar heterosexual. Como señala Natalia Ojeda: "El encierro y el castigo institucionalizado conviven con las alianzas, los amores y las solidaridades, que nos informan acerca de la agencia de estas mujeres, pese al contexto carcelario que insiste en regularlas" (2013, p. 239).

La estadía carcelaria se extendió por el lapso de un año (tres meses en comisaría y nueve en la Unidad N³3) hasta que consiguió el arresto domiciliario, porque la jueza sabía que ella no vendía y que era víctima de violencia. La salida en libertad fue nuevamente difícil, sin trabajo, sin techo y a cargo de los niños/as. En ese entonces recibió hospedaje y ayudas de la ONG “Remar”, a la que define como:

...una ONG cristiana para personas con problemas de abuso, violencia, drogas, en realidad, ayuda a marginados (...) a mí me sanaba mi corazón, me iba poniendo fuerte, muy despacito. 
Mientras tanto, José continuaba detenido con la posibilidad latente de ser excarcelado. Es por ésto que Candela, en pocos meses y a contrarreloj, volvió a la venta ambulante, ahorró el dinero de los subsidios que cobraba por sus hijos/as y el aporte que por única vez entrega el Patronato Bonaerense de Liberados, compró los pasajes en tren a Mar del Plata y migró con todo el "piberío".

C: Puse 80 kilos de ropa en el tren, el Patronato me pagó 3 pasajes, yo pagué 2, y me vine con lo puesto. Caminé, caminé, caminé, caminé y encontré esta casillita, sin nada de nada, dormíamos con los colchones en el piso. (...) Y todavía corro riesgo de que me encuentre y me mate. ¿Quién va a garantizarme que no me va a matar?

F: ¿Tenés miedo?

C: Sí, terrible. Y me comuniqué con varias chicas feministas que saben mi problemática, por lo menos, por si algún día desaparezco.

\section{Discusión}

Las formas en que las mujeres encarceladas "hacen género" (Miller, 2002) no pueden pensarse por fuera de las dinámicas de socialización ligadas a los procesos de criminalización, es decir, de las formas específicas en que son concebidas y tratadas durante su tránsito por los diferentes eslabones del sistema penal. En el caso de Candela, sus primeros contactos se iniciaron desde muy joven, con la detención en el Instituto de Menores de Morón, continuó con sucesivas entradas a comisaría (como detenida primero, y posteriormente como denunciante de violencia de género) y dos ingresos carcelarios, sumado a los numerosos traslados de unidad. Pero también, como es sabido, la circulación por estos espacios, supone la exposición a un conjunto heterogéneo de situaciones de violencia de menor intensidad, pero de carácter sistemático (Perelman \& Tufro, 2017). Prácticas y rutinas extendidas de hostigamiento (Pita, 2019), que resultan constitutivas de las relaciones entre efectivos de las fuerzas de seguridad y habitantes de los barrios pobres o personas en situación de calle.

Por un lado, el "hacer experiencia” en el marco de estos espacios y circuitos, siendo mujer, la situó en otro tipo de prácticas, relaciones y rutinas de violencia institucional, donde fue esencialmente interpelada como un sujeto sexualizado. Por el otro, sus parámetros y significados de género, de ser-mujer, se construyeron sobre la base de umbrales de violencia particularmente exacerbados, cabiendo preguntarse, ¿̇hasta qué punto se puede hablar de un tratamiento de rehabilitación en tanto mujeres, o de re-feminización?, ¿qué tipo de subjetividades y corporalidades se forman al calor de estos circuitos carcelarios o trayectorias de hostigamiento?, ¿y qué tienen que ver con el ideal de feminidad?

En cuanto a la institución familiar, su relato también reconoce allí espacios, prácticas y agentes de hostigamiento. En la descripción de la familia que forma con José, da cuenta de escenas permanentes y cotidianas de violencia de todo tipo, que transcurren en un escenario de feminización de la pobreza, donde Candela ocupa el rol de trabajadora - exclusiva - de los cuidados (Marugán Pintos, 2014) y a la vez, de sostén económico del hogar a costa de la venta ambulante. En este sentido, si la organización (social y económica) de la procreación/crianza 
siguen siendo elementos centrales para la evaluación y definición de lo que es "una familia", podríamos argüir que la experiencia de convivencia con José no se enmarca en el imaginario familiar, o bien, que la idea establecida - heterosexual - de familia soporta o se constituye a través de estas desigualdades y/o violencias.

También es interesante mirar las articulaciones entre violencia de género y violencia institucional, para reinterpretar - en clave interseccional - las (no) actuaciones del Estado frente a la situación de vulnerabilidad de Candela y de sus hijos/as, como pautas sistemáticas y efectivas de vinculación entre el Estado y las mujeres pobres: la pereza judicial reflejada en la falta de investigación, la impunidad de la institución policial plasmada en las innumerables denuncias obsoletas, la negligencia en la implementación de medidas de prevención, etc. Estas acciones, generalmente encuadradas como violencia institucional (para el Sistema Interamericano de Derechos Humanos) en tanto no sólo son consideradas omisiones sino violaciones a derechos fundamentales (Perelman \& Tufro, 2017), podrían igualmente pensarse como mecanismos estatales, y selectivos, de violencia de género.

A su vez, problematizamos la idea de "vida suspendida" o “cancelada” (Martínez, 2020) presentes en investigaciones empíricas con mujeres víctimas de violencia de género, utilizadas para referir a aquellas vidas liminares con la muerte biológica, o donde ha sido obturado el desarrollo de lo que se entiende por vida. En primer lugar, para el caso de las mujeres pobres, entendemos que no se trata de una vida originalmente normal, adulterada por el ejercicio de las violencias, sino vidas e identidades femeninas, que se hacen desde esas mismas condiciones/exclusiones sociales, y violencias variables. Es decir, en las experiencias de ciertas mujeres la llamada violencia de género se rearticula indefectiblemente a otras expresiones de violencia institucional (y viceversa). En segundo lugar, porque hablar de vidas canceladas encierra la imagen de sujetos cancelados, y más allá de su coincidencia - o no - con la realidad concreta de algunas mujeres, consideramos que abona y performa lo femenino como sinónimo de pasividad. En el relato de Candela, su trayectoria, lejos de la suspensión, está movida por un sentido pragmático que le permite gestionar y resolver en pos de su cuidado/defensa y la de sus hijos/as, poniendo en juego aquellos saberes adquiridos mediante y sobre las burocracias penales.

Aquí la agencia, lejos de ser entendida como el ejercicio del libre albedrío, se plantea, parafraseando a Joan Scott (1992), en tanto una condición creada a través de situaciones y estatutos que le son conferidos a los sujetos, quienes se constituyen a través de estas experiencias. La búsqueda es, en definitiva, tratar de comprender las operaciones de lo complejo y cambiante de los procesos discursivos mediante los cuales los sujetos adscriben, resisten o abrazan identidades, siempre contextuales, controvertidas y contingentes.

En este punto, vale señalar que la noción de víctima, incluso de víctima-agente, concebida como aquel sujeto que "hace su agencia en el dolor (...) desde el cuerpo roto y el lenguaje quebrado" (Gatti, 2016, p. 118), continúa asociando dolor - y, por ende, agencia - a una circunstancia o hecho de violencia puntual e identificable, que ha roto o quebrado el curso normal de la vida de una persona de manera difícilmente reversible. Repasando la trayectoria de Candela, ésta condensa otros mecanismos de poder/violencia, no puntuales, sino estructurales, que por ende requieren 
desandar otras estrategias y mecanismos cotidianos, permanentes, de resistencia, pragmáticas de la sobrevivencia, y cuya dimensión política no es comúnmente articulable en sentido oral. "No se tiene una filosofía de este tipo de resistencia, ni un meta-pensamiento de ella: lo que se hace es resistir" (en Abellón, 2014); "se responde con el reflejo de resistir y la voluntad de poner astucia (Martínez, 2018, p. 14). En Candela, se patentiza dicha actitud pragmática en pos de hacer la vida vivible, pero conjugado a un uso estratégico y coyuntural de la victimización, fundado en su conocimiento certero y experiencial del sistema.

Por último, en relación a la institución carcelaria, ésta no representa "ese vacío" de sentido, ese paso definitivo y abismal hacia la degradación, sino que es la instancia de mayor visibilidad, o exoticidad, dentro de un continuum de violencias (Colanzi, 2016). Las correlaciones entre los dispositivos de control afuera/adentro permiten al menos abrir un interrogante sobre el carácter disruptivo de las experiencias carcelarias para ciertas mujeres. A su vez, consideramos necesario relativizar el postulado que entiende a la cárcel como la mera continuación de la violencia que las mujeres padecen durante la infancia, adolescencia y vida anterior a la prisión (Salinas Boldo, 2014), y más aún, como una instancia de pérdida de control sobre el cuerpo, el tiempo y el espacio, donde no sería posible tomar decisiones (Salinas Boldo, 2014).

Si bien coincidimos en que el encarcelamiento reactualiza, generalmente intensificando, ciertas expresiones y prácticas generizantes que se inician en otras instituciones disciplinarias (formales e informales), a la vez, inserta a los cuerpos en condiciones de vida particulares que producen efectos múltiples - nunca compactos - sobre la subjetividad de las mujeres, y que dependerán de los componentes específicos de cada trayectoria femenina. En el caso de Candela, si bien su primera detención fue una estadía prolongada y especialmente conflictiva, la segunda - además de formar parte de su estrategia para salir de la violencia - es enunciada como un período que la encuentra mejor plantada, sabiendo cómo manejarse y forjando vínculos compañeros. En este sentido, representa la posibilidad de insertarse en un grupo, y en lo que ella misma caracteriza como "una familia". Estas grupalidades o familias otras, conforman "redes de apoyo mutuo" (Esteban, 2017), que desafían/amplían la propia categoría de familia asociada a lo doméstico, y a la pareja heterosexual.

\section{Conclusión}

En términos generales, el trabajo permite visualizar esas otras cárceles que atraviesan y condicionan de maneras muy concretas las trayectorias femeninas, y al mismo tiempo, relativizar los sentidos comunes, unívocos y totalizantes sobre el sistema penitenciario, pero también sobre el género de estas mujeres.

Consideramos que el trabajo representa un aporte a los estudios penitenciarios desde una mirada no carcelo-céntrica, en tanto pone de manifiesto que la cárcel no alcanza para explicarse a sí misma, y que es necesario conceptualizar procesos de criminalización de más larga temporalidad que desbordan el encarcelamiento, en tanto comienzan mucho antes y se despliegan aún después de obtenida la libertad. A su vez, interpela a este campo de estudios desde la teoría femininista, al repensar tales procesos mediante la experiencia concreta y singular de una mujer, instando a una pregunta más amplia sobre la dimensión generizante de toda violencia institucional. ¿Cómo afecta 
a los/as jóvenes el hostigamiento policial y judicial en su devenir "hombres” y “mujeres?, ¿cómo se trans-forma su sexualidad y qué pueden (y saben) en tanto cuerpos institucionalizados?, son sólo algunos interrogantes y líneas de indagación que se pueden desprender de un diálogo crítico entre las nociones de violencia institucional y violencia de género.

En cuanto a los Estudios de Género, y fundamentalmente al campo de la criminología feminista, abona, no sólo el análisis de un caso intersectando sus dos principales líneas de trabajo - la selectividad penal/sexual y la violencia familiar - , sino, y ante todo, una perspectiva no victimista que amplifica (y reconfigura) lo que sabemos de las mujeres. De este modo, esperamos haber contribuido a un horizonte epistemológico feminista y antipunitivista, que apunte a desplazar cada vez más el imaginario binario y universalizante de víctimas inocentes, de víctimas perfectas, para el abordaje de la situación de las mujeres pobres, y encarceladas. En su lugar, optamos por la idea de mujeres que se movilizan y resisten de maneras imprevisibles, en tanto sus trayectorias lejos de estar física u ontológicamente detenidas, nos informan de su "fuerza perseverante" (Martínez de la Escalera, 2019), de su constante accionar.

\section{Referencias}

Abellón, P. (2014). María Lugones, una filósofa de frontera que ve el vacío. Revista Mora, (20), 183-189. https://doi.org/10.34096/mora.n20.2340

Aguirre, L. \& Beltrán Savenije, M. A. (2016). Pequeñas conquistas en la adversidad: posibilidades de agencia de mujeres que usan la violencia en Buenos Aires y trabajadoras sexuales en la frontera sur de México. Revista Interdisciplinaria de Estudios de Género, 2(3), 23-49. https://doi.org/10.24201/eg.v2i3.2

Almeda Samaranch, E. (2017). Criminologías feministas, investigación y cárceles de mujeres en España. Papers. Revista Sociológica, 102(2), 151-181. http://dx.doi.org/10.5565/rev/papers.2334

Almeda Samaranch, E., \& Di Nella, D. (2017). Mujeres y cárceles en América Latina. Perspectivas críticas y feministas. Papers. Revista Sociológica, 102(2), 183-214. https://dialnet.unirioja.es/servlet/ articulo? codigo $=5900020$

Antony, C. (2007). Mujeres invisibles: las cárceles femeninas en América Latina. Nueva Sociedad, (208), 7385. https://nuso.org/articulo/las-carceles-femeninas-en-america-latina/

Arduino, I. (2019). Apuntes para renovar la agenda feminista en la criminología. Cuestiones criminales. Cuadernos de Investigación: Apuntes y Claves de Lectura, 2(2), 117-129. https://cutt.ly/NYICCYh

Axat, J. (2008). La prisión preventiva y el confinamiento de niños pobres en institutos de menores "en crisis". Prisma jurídico, 7(2), 321-339. https://doi.org/10.5585/prismaj.v7i2.1396

Beltrán Savenije, M. A. (2010, diciembre 9-10). Criminología feminista. Estado del arte y presencia en Latinoamérica [Ponencia]. VI Jornadas de Sociología de la UNLP, La Plata, Argentina. https://memoria. fahce.unlp.edu.ar/trab_eventos/ev.5515/ev.5515.pdf

Bologna, C. M., Safranoff, A. \& Tiravassi, A. (2019). Contextos de encierro en América Latina: una lectura con perspectiva de género. CELIV, UNTREF. https://celiv.untref.edu.ar/contenidos.html

Butler, J. (2009). Vida precaria. El poder del duelo y la violencia. Paidós.

Cruz Contreras, M. A. (2018). Epistemología feminista y producción de testimonios de mujeres sobre la dictadura en Chile. Prácticas de oficio, 1(21), 65- 75. http://revistas.ungs.edu.ar/index.php/po/article/ view/98

Colanzi, I. C. (2018). Los lazos sexo-afectivos: modos de ejercicio de cuidado de mujeres privadas de libertad. Derecho y Ciencias Sociales, (18), 121-137. https://doi.org/10.24215/18522971eo28 
Colanzi, I. C. (2016, diciembre 5-7). Mujeres encarceladas: el continuum histórico de las violencias institucionales y sus procesos de subjetivación desde una perspectiva de género [ponencia]. IX Jornadas de Sociología de la UNLP, La Plata, Argentina. https://www.memoria.fahce.unlp.edu.ar/ library? $\mathrm{a}=\mathrm{d} \& \mathrm{c}=$ eventos\&d$=$ Jev 9117

Coppa, L. (2019). Feminismo y penalidad como heterotopía. Cuestiones criminales. Cuadernos de Investigación: Apuntes y Claves de Lectura, 2(2),17-42. https://www.lesyc.com/copia-de-c2-roldan

Crenshaw, K. W. (1991). Mapping the Margins: Intersectionality, Identity Politics, and Violence against Women of Color. Stanford Law Review, 43(6), 1.241-1.299. https://doi.org/10.2307/1229039

Daroqui, A., López, A. y Motto, C. (2014). El gobierno del encierro: notas sobre la cuestión carcelaria. En, A. Daroqui (Coord.) Castigar y gobernar. Hacia una sociología de la cárcel. La gobernabilidad penitenciaria bonaerense, (pp. 279-304). CPM y GESPyDH.

Davis, A. (2005). Mujeres, raza y clase. Akal.

Dorlin, E. (2018). Defenderse: Una filosofía de la violencia. Hekht.

Esteban, M. L. (2017). Los cuidados, un concepto central en la teoría feminista: aportaciones, riesgos y diálogos con la antropología. QUADERNS-E DE L'ICA, 2(22), 33-48. https://raco.cat/index.php/ QuadernseICA/article/view/333111

Esteban, M. L. (2004). Antropología encarnada. Antropología desde una misma. Papeles del CEIC, (12), 4-19. https://doi.org/10.1387/pceic.12093

Foucault, M. (2015). Vigilar y castigar. Nacimiento de la prisión. Siglo XXI.

Gago, V. (2019). La potencia feminista. O el deseo de cambiarlo todo. Tinta Limón.

Gago, V. (2014). La razón neoliberal. Economías barrocas y pragmática popular. Tinta Limón.

Garriga Zucal, J. \& Noel, G. D. (2010). Notas para una definición antropológica de la violencia: Un debate en curso. PUBLICAR en Antropología y Ciencias Sociales, 8(9) http://ppct.caicyt.gov.ar/index.php/publicar/ article/view/1191

Gatti, G. (2016). El misterioso encanto de las víctimas. Revista de Estudios Sociales, (56), 117-120. https://doi. org/10.7440/res56.2016.09

Haywood, C., \& Mac an Ghaill, M. (2003). Men and Masculinities. Open University Press Buckingham.

Haraway, D. (1995). Ciencia, Cyborgs y Mujeres. Cátedra.

Herrasti Maciás, M. L. (2010). Mujeres que delinquen. Una encrucijada de la violencia. Trace. Travaux et Recherches dans les Amériques du Centre, (57), 33-47. https://journals.openedition.org/trace/1473

Lozares C. \& Verd, J. M. (2008). La entrevista biográfico-narrativa como expresión contextualizada, situacional y dinámica de la red socio-personal. REDES-Revista hispana para el análisis de redes sociales, 15(6), 95-125. http://dx.doi.org/10.5565/rev/redes.132

Malacalza, L. (2015). Gobernabilidad y violencia: acerca del ejercicio de la maternidad en las cárceles bonaerenses. Derecho y Ciencias Sociales, (12), 154-168. https://revistas.unlp.edu.ar/dcs/article/view/2151

Marcus,S.(2002).Cuerposenlucha, palabrasenlucha:unateoríayunapolítica parala prevencióndelaviolación. Debate feminista, 26, 59-85. https://doi.org/https://doi.org/10.22201/cieg.2594066xe.2002.26.736

Martínez, M. (2020). Living dead: Suspended lives during/after gender violence. Death Studies, 44(11), 718726 https://doi.org/10.1080/07481187.2020.1771855

Martínez, M. (2018). Fuegos de San Telmo. En, E. Dorlin, Defenderse: Una filosofía de la violencia (pp. 11-16). Hekht.

Martínez de la Escalera, A. M. (2019). Crítica de la violencia. Violencia como actividad de inscripción corporal. Acta poética, 40(2), 13-26. http://dx.doi.org/10.19130/iifl.ap.2019.2.853

Marugán Pintos, B. (2014). Trabajo de cuidados. Eunomía. Revista en Cultura de la Legalidad, 7, 215-223. https:// e-revistas.uc3m.es/index.php/EUNOM/article/view/2243 
Miller, J. (2002). The strengths and limits of 'doing gender' for understanding street crime. Theoretical Criminology, 6(4), 433-460. https://doi.org/10.1177/136248060200600403

Núñez Rebolledo, L. (2019). El giro punitivo, neoliberalismo, feminismos y violencia de género. Política y Cultura, (51), 55-81. https://polcul.xoc.uam.mx/index.php/polcul/article/view/1376

Ojeda, N. S. (2013). “Cárcel de mujeres". Una mirada etnográfica sobre las relaciones afectivas en un establecimiento carcelario de mediana seguridad en Argentina. Sociedad y Economía, (25), 237-254. https://cutt.ly/MYIVloG

Perelman, M. y Tufró M. (2017, mayo). Violencia institucional. Tensiones actuales de una categoría política central. CELS. https://cutt.ly/iYIVPrc

Pita, M. V. (2019). Hostigamiento policial o de las formas de la violencia en barrios populares de la Ciudad de Buenos Aires. Relato de una investigación. Desacatos, 6o, 78-93. https://doi.org/10.29340/60.2091

Pitch, T. (2014). La violencia contra las mujeres y sus usos políticos. Revista de Filosofía jurídica y Política, (48), 19-29. https://doi.org/10.30827/acfs.v48io.2778

Rodríguez Alzueta, E. (2015). Circuitos carcelarios: El encarcelamiento masivo-selectivo, preventivo y rotativo en Argentina. En, E. Rodríguez Alzueta y F. Viegas Barriga (Eds.). Circuitos carcelarios. Estudios sobre la cárcel argentina, (pp. 14-59). Ediciones de Periodismo y Comunicación.

Roggeband, C. (2010). The Victim-Agent Dilemma: How Migrant Women Organizations in the Netherlands Deal with a Contradictory Policy Frame. Signs, 35(4), 943-967. https://doi.org/10.1086/651050

Salinas Boldo, C. (2014). Las cárceles de mujeres en México: espacios de opresión patriarcal. Iberóforum. Revista de Ciencias Sociales de la Universidad Iberoamericana, 9(17), 1-27. https://cutt.ly/IYIVZnt

Sánchez, L. J. (2012). De los discursos y los cuerpos sexuales en el campo criminológico y las instituciones penales. En, J. M. Morán Faúndez, M. C. Sgró Ruata y J. M. Vaggione (Coords.). Sexualidades, desigualdades y derechos: reflexiones en torno a los derechos sexuales y reproductivos, (pp. 105-122). Ciencia, Derecho y Sociedad.

Scott, J. W. (1992). “Experience”. En J. Butler y J. W. Scott (eds.): Feminists Theorize the Political, (PP. 22-40). Routledge.

Senatore, A. (2019, agosto 26-30). Ni perversas ni desviadas: Criminalización de la pobreza en clave femenina. [Ponencia]. XIII Jornadas de Sociología, Ciudad Autónoma de Buenos Aires, Argentina. https://cdsa.aacademica.org/ooo-023/686

Stephen, L. (2015). Ser testigo presencial. Acompañando, presenciado, actuando. LASA-OXFAM América 2015 Martin Diskin Memorial Lecture, 46(3), 4-14. https://cutt.ly/XYIV3eJ

Zenobi, D. \& Marentes, M. (2020). Panorama sobre la producción social de las víctimas contemporáneas. En, M. V. Pita \& S. Pereyra (Eds.) Movilización de víctimas y demandas de justicia en la Argentina contemporánea, (pp. 67-100). Teseo Press.

\section{AUTORAS}

María Florencia Actis. Doctora en Comunicación, Especialización en Periodismo, Comunicación Social y Género. Becaria postdoctoral (CONICET) en el Grupo de Estudios sobre Familia, Género y Subjetividades (GEFGS) de la Facultad de Humanidades (UNMDP). Prof. Titular de Posgrado en la Facultad de Periodismo y Comunicación Social (UNLP).

Juliana Inés Arens. Doctoranda en Antropología por la Universidad de Buenos Aires. Magíster en Antropología Social (CIESAS). Becaria Doctoral (CONICET) en el Grupo de Estudios sobre Familia, Género y Subjetividades (GEFGS) de la Facultad de Humanidades (UNMDP). Prof. Adjunta de la asignatura “Fundamentos de la Antropología” en la Universidad Atlántida Argentina. 